ARTICLE

\title{
Porous carbon nanowire array for surface-enhanced Raman spectroscopy
}

\author{
Nan Chen (1) 1,2,12, Ting-Hui Xiao (1) 1,3,12凶 , Zhenyi Luo ${ }^{1}$, Yasutaka Kitahama1, Kotaro Hiramatsu (1) 1,4,5,6, \\ Naoki Kishimoto7, Tamitake Itoh ${ }^{8}$, Zhenzhou Cheng (1) ${ }^{1,9}$ \& Keisuke Goda (1) 1,3,10,11凶
}

Surface-enhanced Raman spectroscopy (SERS) is a powerful tool for vibrational spectroscopy as it provides several orders of magnitude higher sensitivity than inherently weak spontaneous Raman scattering by exciting localized surface plasmon resonance (LSPR) on metal substrates. However, SERS can be unreliable for biomedical use since it sacrifices reproducibility, uniformity, biocompatibility, and durability due to its strong dependence on "hot spots", large photothermal heat generation, and easy oxidization. Here, we demonstrate the design, fabrication, and use of a metal-free (i.e., LSPR-free), topologically tailored nanostructure composed of porous carbon nanowires in an array as a SERS substrate to overcome all these problems. Specifically, it offers not only high signal enhancement $\left(\sim 10^{6}\right)$ due to its strong broadband charge-transfer resonance, but also extraordinarily high reproducibility due to the absence of hot spots, high durability due to no oxidization, and high compatibility to biomolecules due to its fluorescence quenching capability.

\footnotetext{
${ }^{1}$ Department of Chemistry, The University of Tokyo, Tokyo 113-0033, Japan. ${ }^{2}$ School of Chemistry and Chemical Engineering, Beijing Institute of Technology, 100081 Beijing, PR China. ${ }^{3}$ Institute for Quantum Life Science, National Institute for Quantum and Radiological Science and Technology, Chiba 263-8555, Japan. ${ }^{4}$ Research Centre for Spectrochemistry, The University of Tokyo, Tokyo 113-0033, Japan. ${ }^{5}$ PRESTO, Japan Science and Technology Agency, Saitama 332-0012, Japan. ${ }^{6}$ Kanagawa Institute of Industrial Science and Technology, Kanagawa 243-0435, Japan. ${ }^{7}$ Department of Chemistry, Tohoku University, Sendai 980-8578, Japan. ${ }^{8}$ Health and Medical Research Institute, National Institute of Advanced Industrial Science and Technology, Takamatsu 761-0395, Japan. ${ }^{9}$ School of Precision Instruments and Opto-electronics Engineering, Tianjin University, 300072 Tianjin, PR China. ${ }^{10}$ Institute of Technological Sciences, Wuhan University, 430072 Hubei, PR China. ${ }^{11}$ Department of Bioengineering, University of California, Los Angeles, CA 90095, USA. ${ }^{12}$ These authors contributed equally: Nan Chen, Ting-Hui Xiao. ${ }^{凶}$ email: xiaoth@chem.s.u-tokyo.ac.jp; goda@chem.s.u-tokyo.ac.jp
} 
S urface-enhanced Raman spectroscopy (SERS) $)^{1-7}$ is an effective tool for vibrational spectroscopy by virtue of its ability to provide several orders of magnitude higher sensitivity than inherently weak spontaneous Raman scattering by exciting localized surface plasmon resonance (LSPR) on metal substrates. Unfortunately, the benefit of SERS is compromised by its poor reproducibility, uniformity, biocompatibility, and durability $^{8-12}$ due to its dependence on hot spots ${ }^{13-15}$ for high enhancement factors from aggregates of metal nanoparticles ${ }^{16,17}$ or engineered metal nanostructures $4,14,15$, the generation of large photothermal heat on the metal surface that causes detrimental effects to biomolecules (e.g., heat-induced protein denaturation $)^{12,18}$, and the oxidization of the metal surface ${ }^{11}$. While various efforts have been made to overcome these problems, it still remains challenging to develop a SERS substrate that simultaneously achieves high sensitivity, uniformity, and reproducibility since it requires a high density of hot spots with uniform enhancement factors ${ }^{8}$. The problems are even more prominent for biomedical use because the reliability of the SERS substrate is the key to extracting meaningful information from SERS data, quantitatively analyzing and interpreting it, and reaching accurate conclusions ${ }^{8}$. In recent years, nonmetallic materials such as silicon and germanium nanostructures ${ }^{19-21}$, two-dimensional materials (e.g., graphene, $\mathrm{MoS}_{2}$, and h-BN) ${ }^{11,22,23}$, and semiconducting metal oxides ${ }^{24,25}$ have been proposed as alternative SERS substrates to circumvent the above predicament. Different from the metal substrates that mainly rely on LSPR, their signal enhancement originates from structural resonance ${ }^{20,21}$ or charge-transfer resonance ${ }^{23,24}$ and has been demonstrated with decent enhancement factors of up to five orders of magnitude $20,21,23,24$. However, the poor reproducibility remains a major challenge while these substrates partly address the biocompatibility issue. This is due to their inherent photocatalytic activity and the toxicity of their substrate material to biomolecules $^{26,27}$.

In this Article, we take a different approach from the metal, semiconducting, and dielectric substrates and demonstrate the design, fabrication, and use of a LSPR-free, topologically tailored nanostructure composed of a two-dimensional array of porous carbon nanowires as an effective substrate for highly sensitive, biocompatible, and reproducible SERS (Supplementary Table 1, Supplementary Note 1). Specifically, the porous carbon nanowire array (PCNA) substrate provides not only high signal enhancement $\left(\sim 10^{6}\right)$ due to its strong broadband charge-transfer resonance for large chemical enhancement (as opposed to electromagnetic enhancement in traditional SERS based on metal substrates), but also extraordinarily high reproducibility or substrate-to-substrate, spot-to-spot, sample-to-sample, and time-totime consistency in SERS spectrum (which is not possible with traditional SERS) due to the absence of "electromagnetic hot spots" by making the entire surface of the substrate "chemically hot", high durability due to no oxidization, and high compatibility to biomolecules due to its fluorescence quenching capability. We experimentally demonstrate these excellent properties with various molecules such as rhodamine 6G (R6G), $\beta$-lactoglobulin, and glucose. Our PCNA substrate overcomes the decades-old problems of conventional SERS by simultaneously achieving high sensitivity, uniformity, reproducibility, biocompatibility, and durability, thereby leading to high reliability for practical use, especially in the areas of analytical chemistry, pharmaceutical science, food science, forensic science, and pathology where inconsistent or non-reproducible SERS spectra have been problematic.

\section{Results}

Synthesis and characterization of the PCNA. The synthesis and material properties of the PCNA are described as follows and shown in Fig. 1a through Fig. 1d (Supplementary Notes 2-4). First, a polypyrrole (PPy) nanowire array (PNA) is prepared through a conventional template-assisted electropolymerization method $^{28}$ with an anodized aluminum oxide (AAO) template (Supplementary Fig. 1). Second, a working electrode is interchanged with a counter electrode. Then, the PNA undergoes an electrical degradation process in a high-temperature dimethyl sulfoxide (DMSO) solution containing sulfur clusters under an oppositely applied voltage to form a large number of nanopores in each PPy nanowire, which transforms into a porous polypyrrole nanowire array (PPNA) and effectively increases the specific surface area (SSA) and roughness. Finally, a carbonization process at an elevated temperature is applied to the PPNA to generate a SERS-active PCNA (Supplementary Figs. 2, 3, Supplementary Note 5). Each porous nanowire of the PCNA has an average diameter of $140 \mathrm{~nm}$ and an average length of $15 \mu \mathrm{m}$ after the carbonization (Fig. 1a, Supplementary Fig. 4, Supplementary Note 6), which is in agreement with those of the AAO template. An enlarged scanning electron microscope (SEM) image of the PCNA reveals that numerous holes with an average diameter of about $50 \mathrm{~nm}$ are distributed on the PCNA, resulting in fractal nanostructures with a high SSA (Supplementary Figs. 5, 6, Supplementary Notes 7,8 ). In addition, as shown in Fig. 1b, results from our Raman spectroscopy of the PPNA and PCNA indicate that all the characteristic Raman peaks of the PPNA at 870, 930, 1050 , and $1246 \mathrm{~cm}^{-1}$ disappeared from the PCNA after the carbonization, as expected. As shown in Fig. 1c, results from our current-voltage (I-V) curve measurements of the PPNA and PCNA indicate that the porous carbon nanomaterial performs as a semiconductor since the carbonization treatment significantly increases its conductivity (Supplementary Figs. 7, 8, Supplementary Notes 9, 10). Moreover, results from our energy-dispersive X-ray spectroscopy (EDS) of the PPNA and PCNA indicate that the composition ratio of carbon significantly increased after the carbonization process as shown in Fig. 1d.

High reproducibility and fluorescence quenching of the PCNA. To demonstrate the high adsorptivity and reproducibility of the PCNA as a SERS substrate, we performed SERS of R6G (a highly fluorescent rhodamine family dye) on the PCNA substrate. For comparison, we obtained Raman spectra of R6G molecules adsorbed on silicon, PNA, carbon nanowire array (CNA), and PCNA substrates under the same conditions (a $10 \mu \mathrm{M}$ deionized water solution of R6G molecules, an integration time of $30 \mathrm{~s}$, continuous-wave laser illumination with $1 \mathrm{~mW}$ at $785 \mathrm{~nm}$ ), as shown in Fig. 2a (Supplementary Fig. 9, Supplementary Table 2, Supplementary Note 11). In comparison to the other substrates, the PCNA exhibits the highest Raman signal intensity, indicating its high adsorptivity (i.e., large surface-to-volume ratio), which originates from its porous nanowire morphology and carbonbased material. Moreover, to evaluate the sensitivity of the PCNA substrate, we obtained SERS spectra of R6G molecules at reduced concentrations on the PCNA substrate (Fig. 2b). It is evident from the figure that the lowest detectable concentration of the PCNA substrate for R6G molecules is about $10 \mathrm{nM}$. It is important to note that the use of carbon quenched the fluorescent property of R6G molecules, even including anti-Stokes fluorescence in the case of excitation at $785 \mathrm{~nm}$ caused by the thermal excitation (Boltzmann distribution) that allows the excitation photon energy to be smaller than the energy gap between the ground state and an excited state ${ }^{29}$, indicating the ability of the PCNA substrate to analyze biomolecules which are mostly fluorescent (Supplementary Fig. 10, Supplementary Note 12). Similar SERS measurements were performed on DMSO to verify the high sensitivity of the PCNA substrate (Supplementary 
a
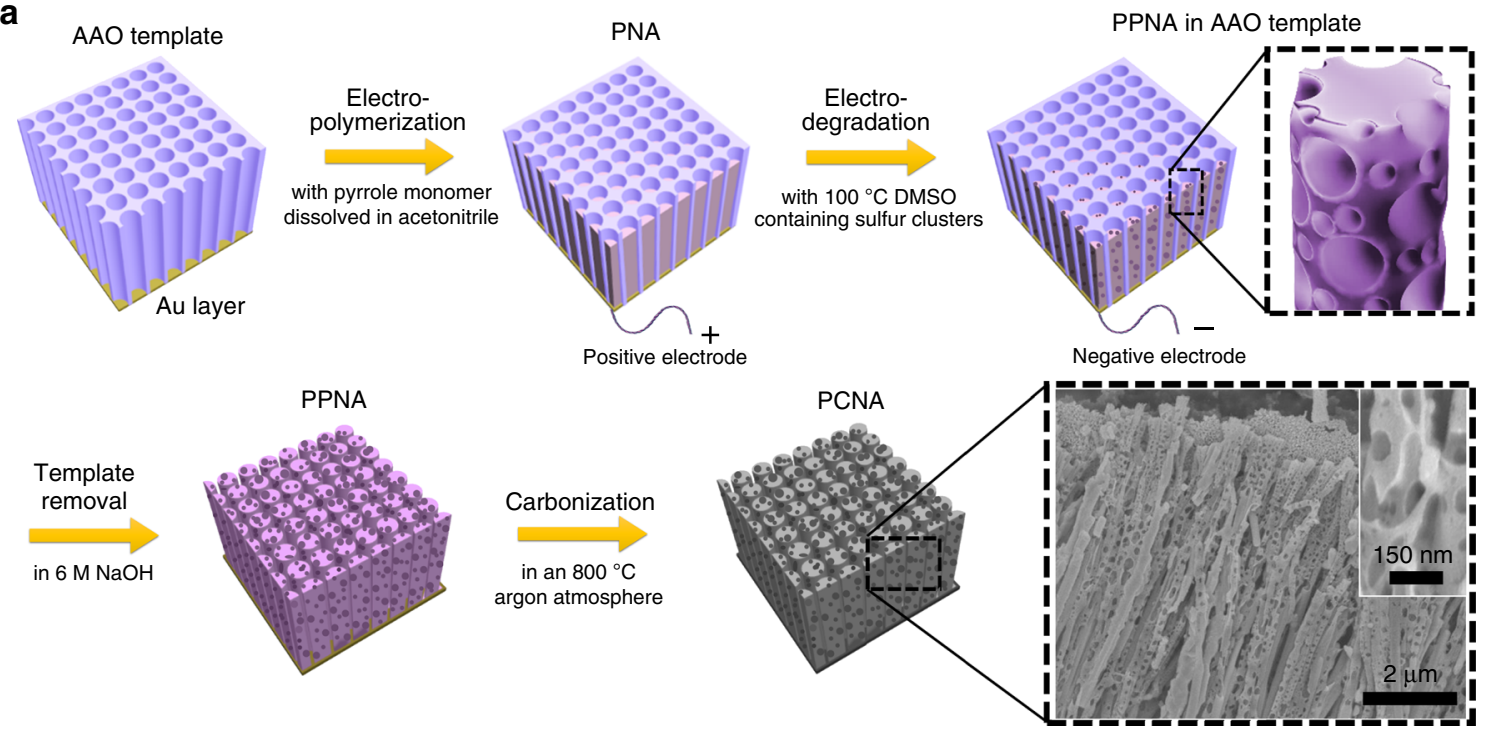

b

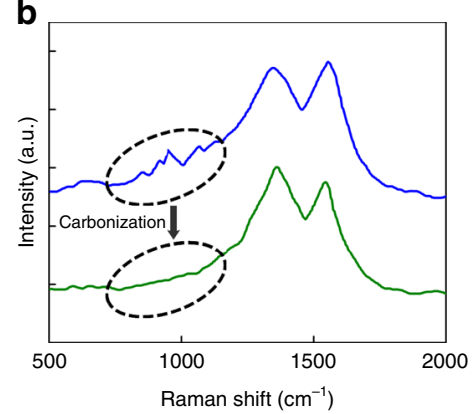

c

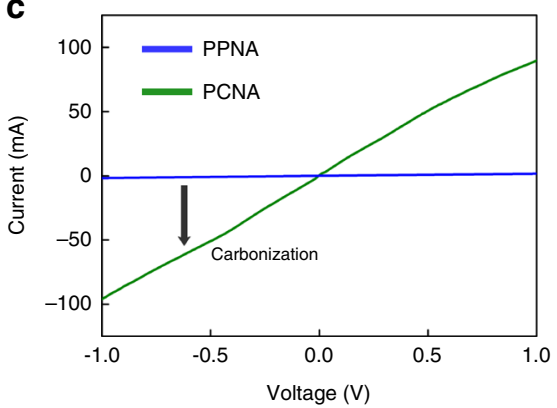

d

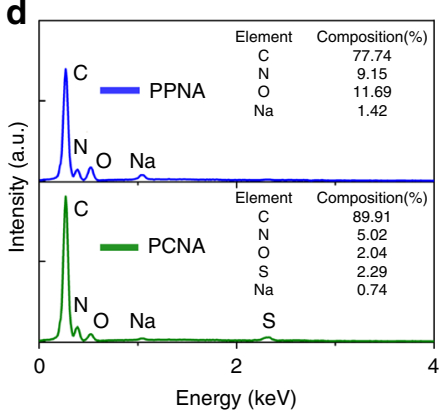

Fig. 1 Synthesis and characterization of the PCNA. a Steps for synthesizing the PCNA. The bottom right inset shows an SEM image of the PCNA and an enlarged SEM image of a single porous carbon nanowire. $\mathbf{b}$ Raman spectra of the PPNA and PCNA. After the carbonization process, all the characteristic Raman peaks of the PNA at $870,930,1050$, and $1246 \mathrm{~cm}^{-1}$ disappeared as evident in the PCNA spectrum. $\mathbf{c}$ I-V curve measurements of the PPNA and PCNA. In the measurements, the length and contact area of the porous PPy nanowires and porous carbon nanowires are about $15 \mu \mathrm{m}$ and $0.5 \mathrm{~mm}{ }^{2}$, respectively. The conductivity of the substrate was significantly increased after the carbonization process, indicating the semiconducting property of the PCNA. d EDS spectra of the PPNA and PCNA. The composition ratio of carbon was significantly increased after the carbonization process.

Fig. 11, Supplementary Note 13). As shown in Fig. 2c, the PCNA substrate shows a monotonically increasing relation between the R6G concentration and signal intensity at 1185, 1309, 1361, 1507, and $1650 \mathrm{~cm}^{-1}$. Furthermore, in order to assess the substrate-tosubstrate consistency of the PCNA substrate, we carried out a measurement reproducibility test on 20 different PCNA substrates. As shown in Fig. $2 \mathrm{~d}$, the differences in the relative intensities of the Raman peaks at 1185, 1309, 1361, 1507, and $1650 \mathrm{~cm}^{-1}$ between all of the substrates are with a standard deviation of $5.7 \%$, indicating the high reliability of the PCNA substrate (Supplementary Note 14). It is important to mention that the Raman intensity of the PCNA substrate (Fig. 1b) does not interfere with the enhanced Raman spectra of probed molecules since it is much weaker than them and its characteristic peaks are very broad and submerged by them (Supplementary Fig. 12).

High sensitivity and uniformity of the PCNA. To demonstrate the highly sensitive detection of biomolecules, we conducted SERS of $\beta$-lactoglobulin (the major whey protein of cow and sheep's milk) on the PCNA substrate. With an integration time of $300 \mathrm{~s}$ and an excitation illumination of $2 \mathrm{~mW}$ at $785 \mathrm{~nm}$, we measured a spontaneous Raman spectrum of $\beta$-lactoglobulin powder with a mass fraction (Mf) of $100 \%$ on a bare silicon substrate as the ground truth (Fig. 3a, Supplementary Fig. 9,
Supplementary Table 2, Supplementary Note 11). Then, by decreasing the integration time to $1 \mathrm{~s}$ and the Mf to $0.4 \%$, we obtained the Raman spectrum of $\beta$-lactoglobulin molecules with a similar Raman signal intensity on the PCNA substrate. As the ratio of the number of molecules of a $\beta$-lactoglobulin solution to that of the $\beta$-lactoglobulin powder in the probed volume is approximately equal to their Mf ratio (Supplementary Table 3, Supplementary Note 15), we used the Mf ratio to calculate theSERS enhancement, based on which the average enhancement factor in the probed volume (not the local enhancement factor on the surface of each porous carbon nanowire) was found to be $(300 \mathrm{~s} / 1 \mathrm{~s}) \times(100 \% / 0.4 \%) \times(60,000 / 2000)=\sim 10^{6}$ for $\beta$-lactoglobulin. It is important to note that the characteristic Raman peaks of $\beta$-lactoglobulin molecules on the PCNA substrate are well distinguishable and consistent with those of the ground truth (with small differences which may be attributed to the different adsorption orientations of the biomolecules on the silicon and PCNA substrates ${ }^{5}$ ), which is verified by our peak assignment (Supplementary Fig. 9, Supplementary Table 2, Supplementary Note 11), indicating the high biocompatibility of the PCNA substrate. For comparison, we also acquired the Raman spectra of $\beta$-lactoglobulin molecules on a silicon substrate and a commercial metal SERS substrate (sliver-gold hybrid substrate, SERSitive Co.) under the same conditions, as shown in Fig. 3a and Supplementary Fig. 13. On the silicon substrate, no 
a
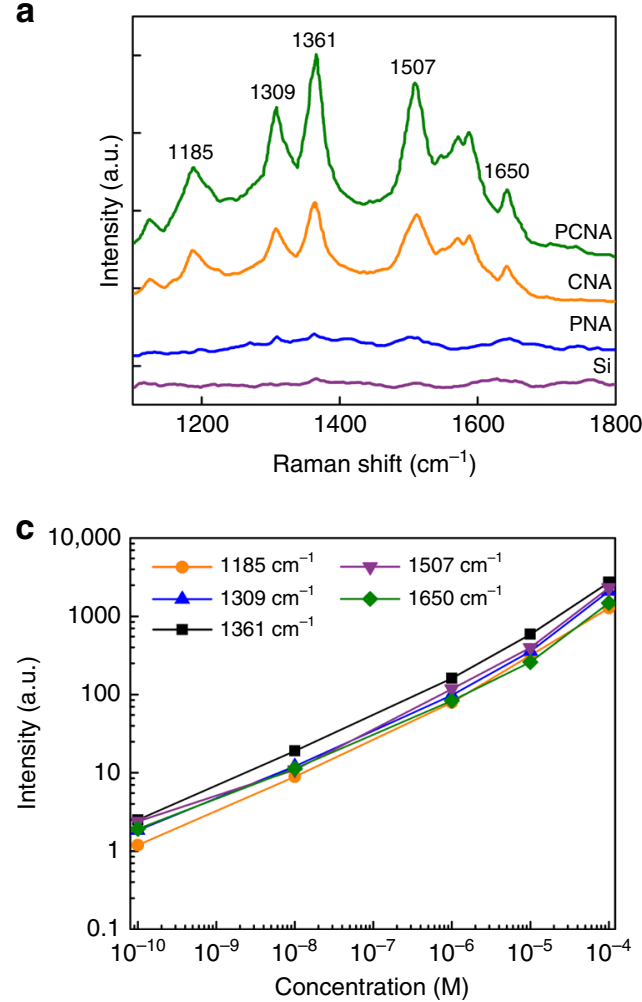

b

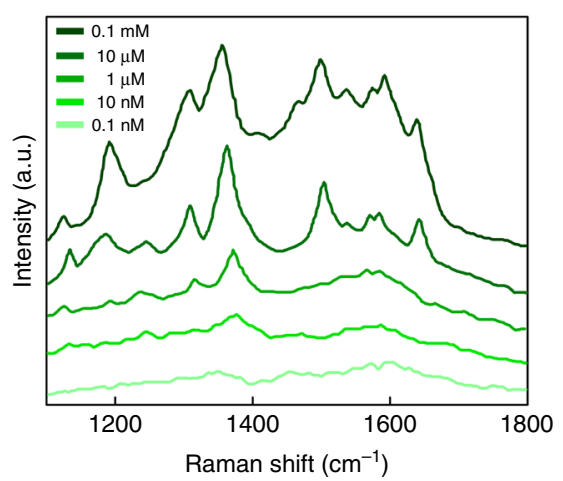

d

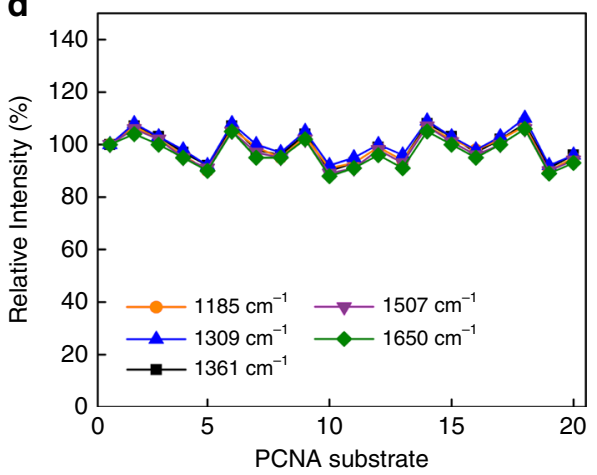

Fig. 2 SERS of R6G on the PCNA substrate. a Measured Raman spectra of R6G molecules at a concentration of $10 \mu \mathrm{M}$ on the silicon (red), PNA (blue), CNA (orange), and PCNA (green) substrates for an integration time of $30 \mathrm{~s}$ with an excitation power of $1 \mathrm{~mW}$ at $785 \mathrm{~nm}$ after cleaning the substrates. b Measured Raman spectra of R6G molecules at different concentrations adsorbed on the PCNA substrate for an integration time of $30 \mathrm{~s}$ with an excitation power of $1 \mathrm{~mW}$ at $785 \mathrm{~nm}$. c Intensities of the Raman peaks at different concentrations at 1185, 1309, 1361, 1507, and $1650 \mathrm{~cm}^{-1}$. The detection limit of the PCNA substrate for R6G molecules is about $10 \mathrm{nM}$. d SERS reproducibility measurements of R6G molecules on different PCNA substrates. The differences in the relative intensities of the Raman peaks at 1185, 1309, 1361, 1507, and $1650 \mathrm{~cm}^{-1}$ between 20 different substrates are within a standard deviation of $5.7 \%$.

characteristic Raman peaks were visible due to the absence of the SERS enhancement whereas on the metal substrate, the Raman spectrum was enhanced, but with 10 times weaker than on the PCNA substrate. Also, the characteristic Raman peaks of $\beta$ lactoglobulin molecules on the metal substrate do not agree with those of the ground truth obtained from the silicon substrate (Fig. 3a). Finally, to assess the spot-to-spot consistency of the PCNA substrate in Raman signal intensity, we conducted SERS mapping of $\beta$-lactoglobulin on the PCNA substrate at two characteristic Raman peaks of the molecule (999 and $1447 \mathrm{~cm}^{-1}$ ) on both large and small scales (Fig. 3b). As shown in Fig. 3c, the PCNA substrate has a coefficient of variation $(\mathrm{CV})$ of $<7.8 \%$ on average, which is much smaller than that of the conventional metal substrate (Supplementary Fig. 14, Supplementary Note 16). These results firmly demonstrated that the PCNA substrate would be an excellent platform for reliable trace detection of proteins which are typically vulnerable to heat and are difficult to probe on metal substrates.

High consistency and durability of the PCNA. To demonstrate the sample-to-sample consistency of the PCNA substrate in the enhancement factor, we conducted SERS of glucose (a wellknown biomarker for detecting diabetes ${ }^{30}$, which has been a challenging molecule for undistorted SERS detection. Figure $3 \mathrm{~d}$ shows the Raman spectra of a deionized water solution of glucose molecules on silicon and PCNA substrates under light excitation at $785 \mathrm{~nm}$, with the former used as a ground truth (Supplementary Fig. 9, Supplementary Table 2, Supplementary Note 11). On the PCNA substrate, all the characteristic Raman peaks of glucose molecules were clearly identified and distinguished even at a low Mf of $0.1 \%$, corresponding to a concentration of $5.6 \mathrm{mM}$ (which agrees with the typical glucose concentration of $3-7 \mathrm{mM}$ in the blood of healthy people as opposed to $10-20 \mathrm{mM}$ in the blood of diabetes patients ${ }^{31}$. Similar to that of $\beta$-lactoglobulin, we used the Mf ratio of glucose to calculate the SERS enhancement (Supplementary Table 3, Supplementary Note 15), based on which the comparison of the Raman spectra on the silicon and PCNA substrates yields an enhancement factor of $(300 \mathrm{~s} / 1 \mathrm{~s}) \times(100 \% /$ $0.1 \%) \times(1600 / 500)=\sim 10^{6}$, which is consistent with the enhancement factor of the $\beta$-lactoglobulin measurements above. Importantly, even with this high signal enhancement, the locations of the measured characteristic Raman peaks of glucose molecules agree well with those of the ground truth (while each Raman peak has a different chemical enhancement), which is attributed to the high biocompatibility and photothermal stability of the PCNA substrate. Furthermore, to show the time-to-time consistency of the PCNA substrate, we performed SERS measurements of glucose under the same conditions every hour. As shown in Fig. 3e, the Raman spectrum of glucose molecules is temporally stable with negligible deterioration (e.g., no oxidization) indicated by a small CV value of $15.1 \%$ for at least $4 \mathrm{~h}$ as opposed to many metal SERS substrates that cannot be used for more than $1 \mathrm{~h}$ since they are easily oxidized in the air (Supplementary Figs. 15, 16, Supplementary Note 17), further demonstrating the high reliability of the PCNA substrate.

Theory and experimental verification. The SERS signal enhancement of the PCNA is mainly attributed to the chemical 
a

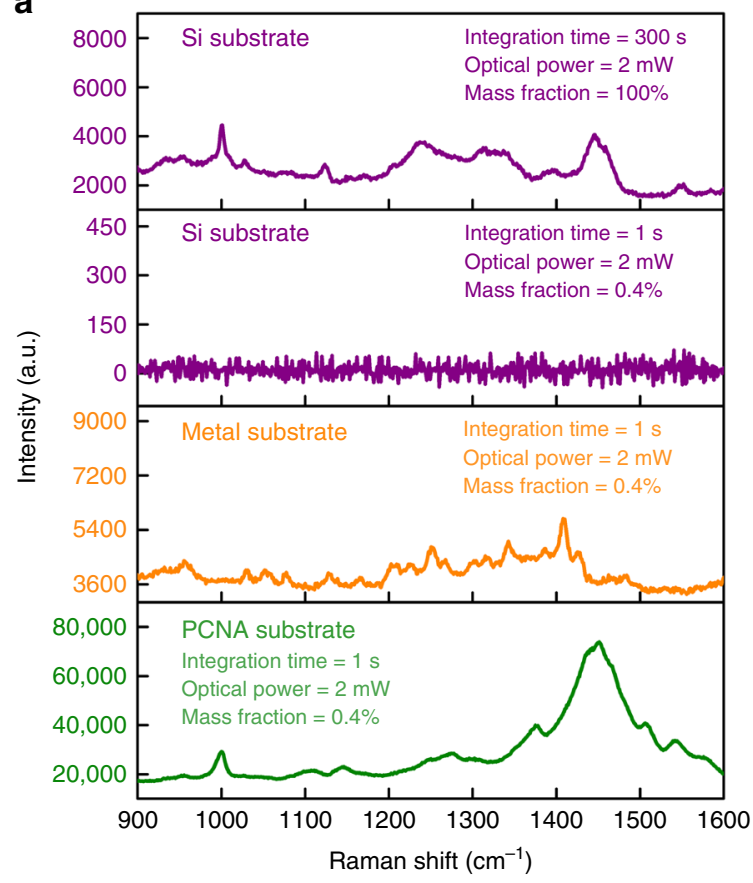

d

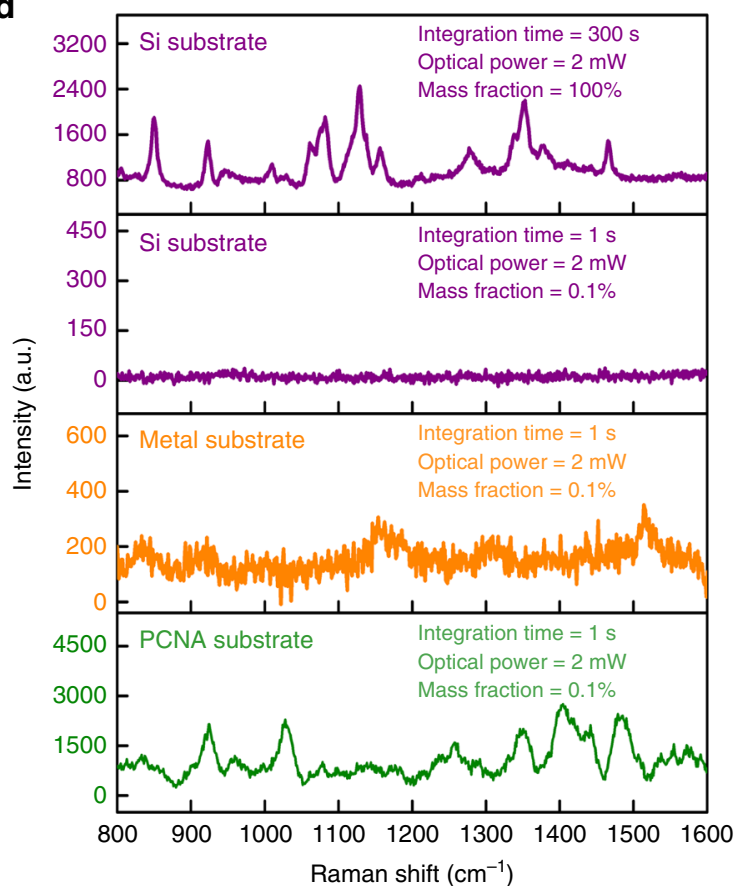

b

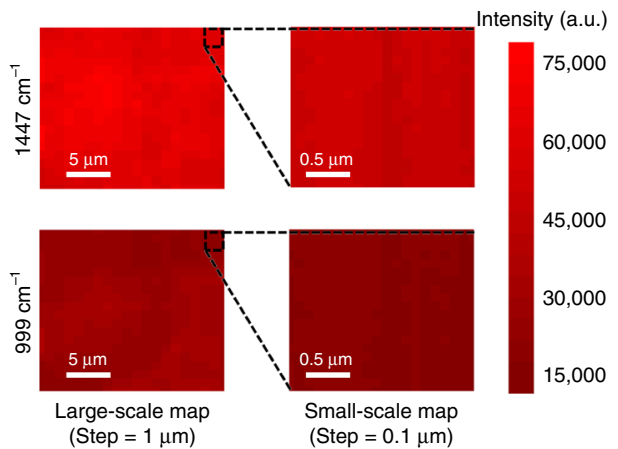

C

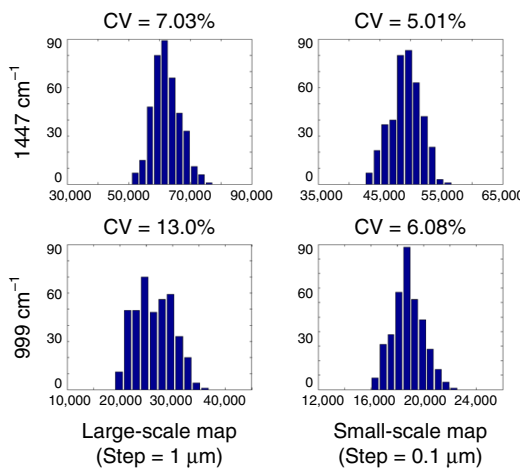

e

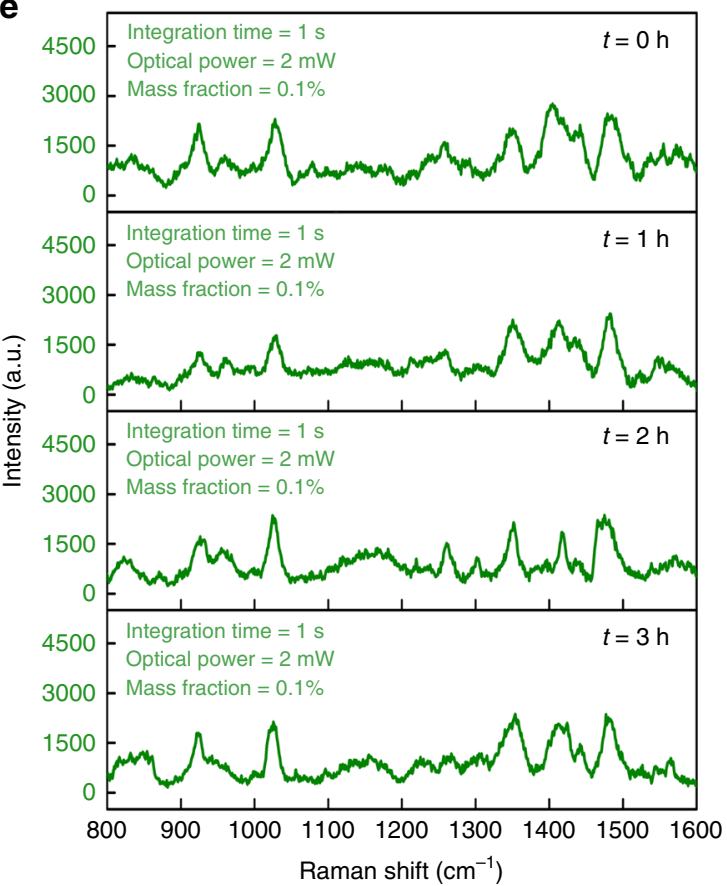

Fig. 3 SERS of biomolecules ( $\beta$-lactoglobulin and glucose) on the PCNA substrate. a Measured Raman spectra of $\beta$-lactoglobulin molecules on the silicon, PCNA, and commercial metal substrates. The Raman signal intensity of $\beta$-lactoglobulin molecules on the PCNA substrate is 10 times higher than that on the metal substrate. $\mathbf{b}$ SERS maps of $\beta$-lactoglobulin on the PCNA substrate, showing high surface homogeneity in enhancement factor at two characteristic Raman shifts of the molecule on both large and small scales with a step size of 1 and $0.1 \mu \mathrm{m}$, respectively. $\mathbf{c}$ Histograms of the enhancement factors on the large and small scales. d Measured Raman spectra of glucose molecules on the silicon and PCNA substrates. e Time-to-time consistency of the PCNA substrate in the Raman spectrum of glucose. Small hour-to-hour fluctuations in the Raman spectrum indicate high reproducibility and biocompatibility.

mechanism $(\mathrm{CM})$ for the following reasons. First, carbon provides a high charge-transfer efficiency ${ }^{23}$, which greatly increases its Raman-scattering cross-section. Figure 4a shows an energy level diagram (obtained by our theoretical analysis based on density functional theory with Gaussian16) that indicates the molecular orbitals of a test molecule $(\mathrm{R} 6 \mathrm{G})$ on a carbon sheet that approximates the surface of the PCNA (Supplementary Fig. 17, Supplementary Note 18). The diagram also indicates two chargetransfer pathways from the highest occupied molecular orbital (HOMO) to the PCNA-R6G hybrid states, enabling the R6G 
a
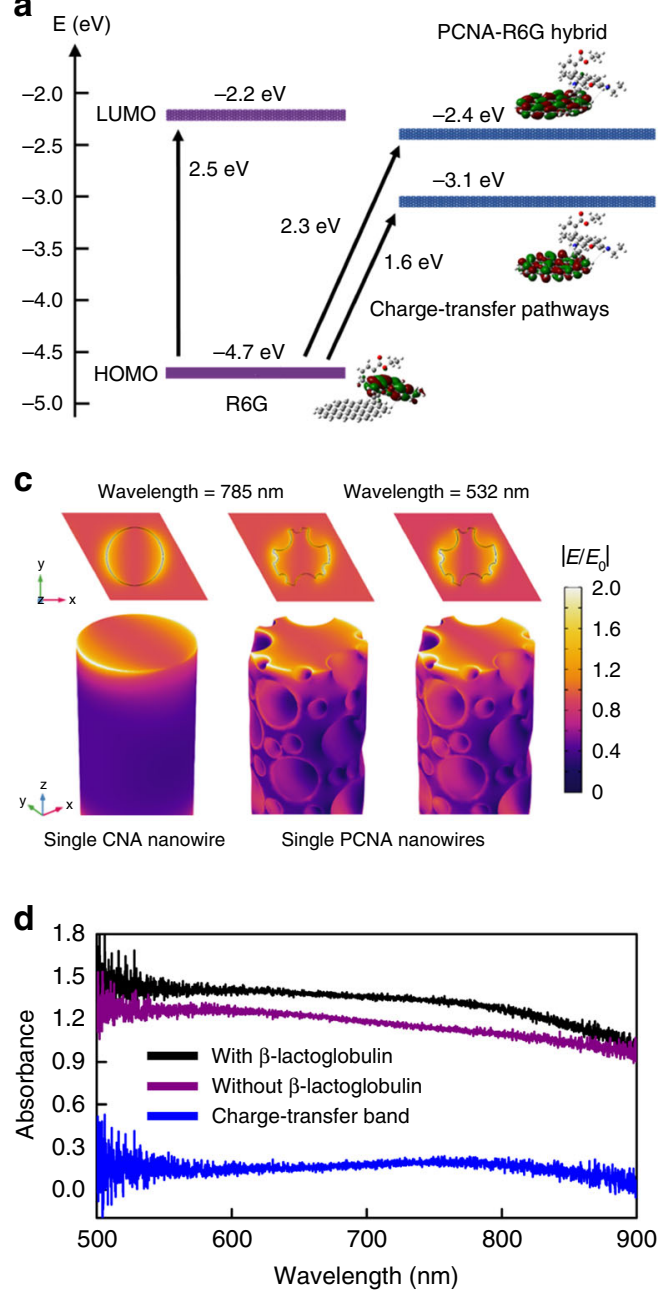

e

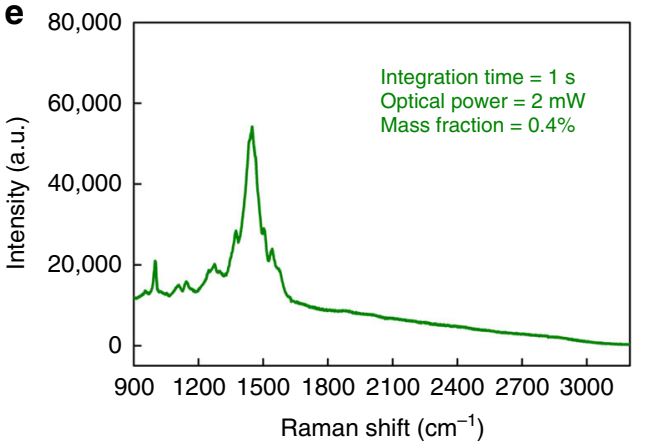

b

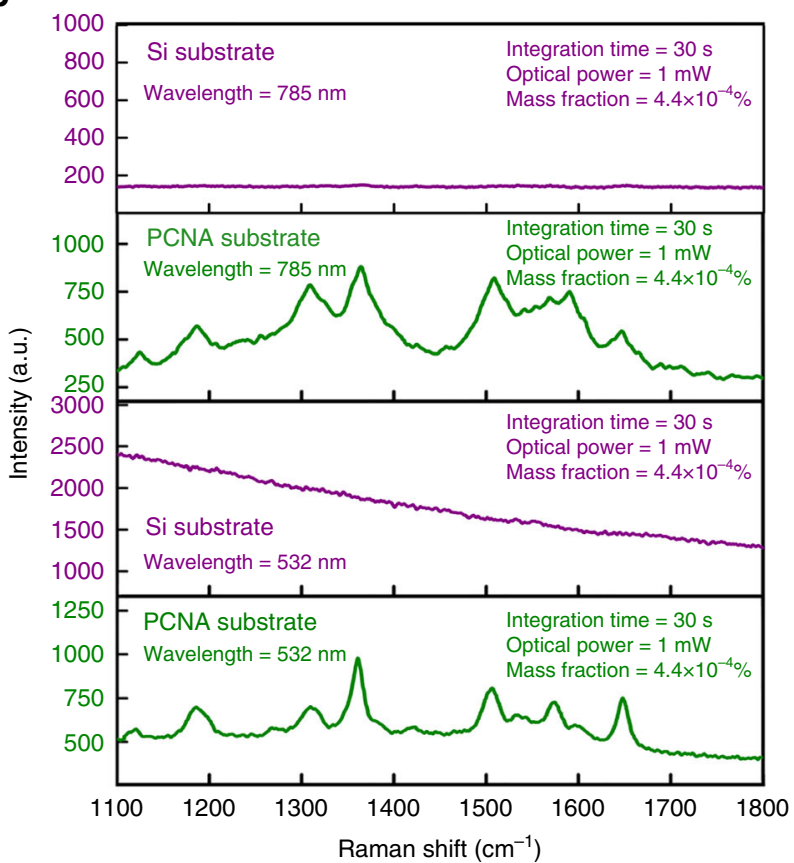

f

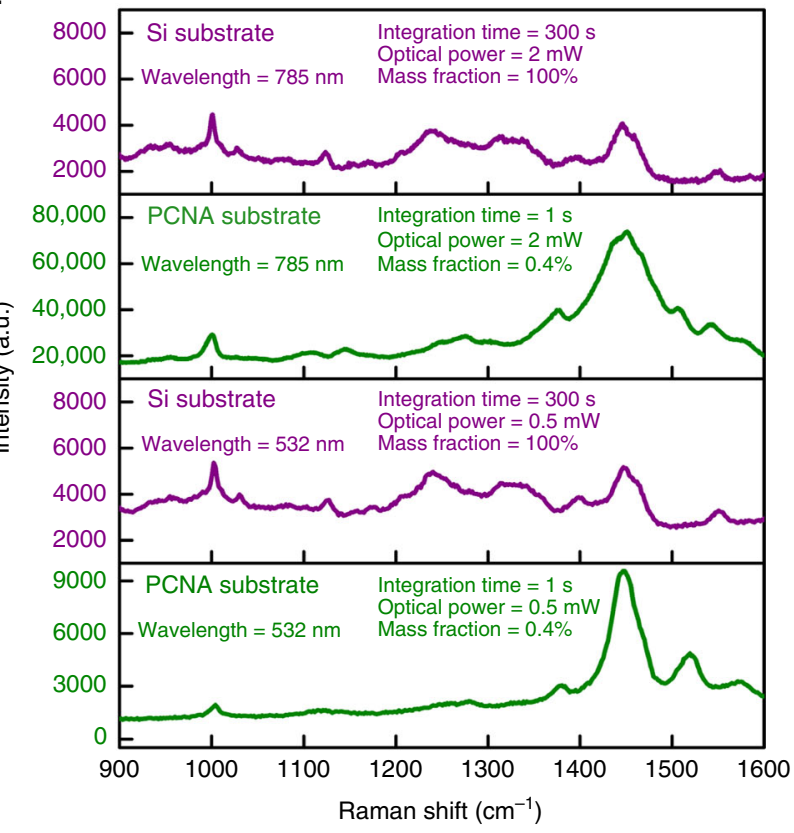

Fig. 4 Theory and experimental verification of the CM as the dominant effect on the PCNA substrate. a Energy level diagram (obtained by our theoretical analysis based on density functional theory with Gaussian16) that shows the molecular orbitals of a test molecule (R6G) on a carbon sheet that approximates the surface of the PCNA. The diagram shows two charge-transfer pathways from the HOMO to the PCNA-R6G hybrid states, enabling the R6G molecule on the surface of the PCNA to resonantly excited at the wavelengths of $785 \mathrm{~nm}(1.58 \mathrm{eV})$ and $532 \mathrm{~nm}(2.33 \mathrm{eV})$, whereas the R6G molecule alone cannot be resonantly excited at $785 \mathrm{~nm}$ due to the absence of excited states between the HOMO and the LUMO and can be resonantly excited at $532 \mathrm{~nm}$, but with a very strong fluorescence background that obscures the Raman spectrum. b Raman spectra of R6G on the silicon and PCNA substrates at excitation wavelengths of 532 and $785 \mathrm{~nm}$. c Comparison in electric field magnitude distribution between the single CNA nanowire and the single PCNA nanowire to visualize the small contribution of the EM. $\mathbf{d}$ Absorption spectrum of the PCNA substrate with and without $\beta$-lactoglobulin on it and the charge-transfer band obtained by taking the difference of the two absorption spectra. e Raman spectrum of $\beta$-lactoglobulin up to the high Raman shift region, showing no appreciable peaks of overtones and combination bands. $\mathbf{f}$ Raman spectra of $\beta$-lactoglobulin at different excitation wavelengths of 532 and $785 \mathrm{~nm}$. 
molecule on the surface of the PCNA to resonantly excited at the wavelengths of $785 \mathrm{~nm}(1.58 \mathrm{eV})$ and $532 \mathrm{~nm}(2.33 \mathrm{eV})$, whereas the $\mathrm{R} 6 \mathrm{G}$ molecule alone cannot be resonantly excited at $785 \mathrm{~nm}$ due to the absence of excited states between the HOMO and the lowest unoccupied molecular orbital (LUMO) and can be resonantly excited at $532 \mathrm{~nm}$, but with a very strong fluorescence background that obscures the Raman spectrum. These theoretically predicted resonant Raman scattering effects, which account for the extraordinary SERS enhancements at the excitation wavelengths of 532 and $785 \mathrm{~nm}$, are in good agreement with our experimental results as shown in Fig. 4b. Second, H, N, and S atoms in the hydroxyl group that are not completely removed after the carbonization (Fig. 1d) further promote the chargetransfer between the substrate and molecules ${ }^{15}$, contributing to the SERS enhancement. Third, based on our finite element method simulation (Fig. 4c), the largest electric field enhancement factor localized at the lateral edges of each PCNA nanowire is only about 2 , corresponding to a small average enhancement factor of $\sim 1.8$ (Supplementary Fig. 18). Also, the small difference between the electric field magnitude distributions at the excitation wavelengths of 532 and $785 \mathrm{~nm}$ shows the absence of strong structural resonance, indicating a small electromagnetic contribution to the overall SERS enhancement factor.

To further verify this theory, we performed the following experiments. First, we measured the absorption spectra of the PCNA substrate with and without $\beta$-lactoglobulin on it as shown in Fig. 4d. The broad bandwidth of the absorption spectrum excludes the electromagnetic mechanism (EM) which normally possesses a narrow bandwidth due to its structural resonance. Figure $4 \mathrm{~d}$ also shows the charge-transfer band obtained by taking the difference of the two absorption spectra (Supplementary Fig. 19). The broad charge-transfer band that covers the excitation wavelength $(785 \mathrm{~nm})$ indicates an electronic band essential for the efficient charge-transfer resonance. Second, we measured the Raman spectrum of the same molecule up to the high Raman shift region (up to $3200 \mathrm{~cm}^{-1}$ ) which covers overtones and combination bands of the molecule as shown in Fig. 4e. No peaks of overtones and combination bands were observed in the region. As overtones and combination bands are nonlinear optical effects whose magnitudes are determined by the electric field magnitude and normally evident in EM-based SERS, the absence of their peaks in this region excludes the EM, further verifying the $\mathrm{CM}$ as the dominant effect of the Raman enhancement. Third, we measured the Raman spectrum of $\beta$ lactoglobulin at different excitation wavelengths (785 and 532 $\mathrm{nm})$. As shown in Fig. 4f, enhancement factors of $\sim 10^{6}$ and $\sim 10^{5}$ at the excitation wavelengths of 785 and $532 \mathrm{~nm}$, respectively, were observed, verifying the broadband $\mathrm{CM}$ enhancement of the PCNA substrate.

\section{Discussion}

By virtue of the combined merits of the high reproducibility and excellent biocompatibility of the PCNA substrate, the current enhancement factor of $10^{6}$ is sufficient for practical use for Raman spectroscopy of biomolecules at low concentrations as it is comparable to or even higher than the enhancement factor of commercial metal SERS substrates. A higher SERS enhancement factor can presumably be achieved by optimizing the composition (e.g., types and doping levels of dopants) and structure (e.g., porous nanowire morphology) of the PCNA substrate, employing an excitation laser whose wavelength is optimal for the $\mathrm{CM}$ as the enhancement factor depends on the probed molecule (e.g., via a wavelength-tunable laser), or improving the theory of the $\mathrm{CM}$ for a better understanding of its underlying principles. With these improvements, PCNA-based SERS is expected to enable a wider range of biomedical applications such as quantitative analysis of chemical bonds in proteins, accurate evaluation of glucose in blood for diabetes detection, and trace detection of toxic substances in food and water.

\section{Data availability}

The source data supporting the findings of this study are available at https://doi.org/ 10.5281/zenodo.3994312, and are also available from the corresponding authors upon reasonable request.

\section{Code availability}

All codes used for analysis of this study are available from the corresponding authors upon reasonable request.

Received: 10 August 2020; Accepted: 26 August 2020; Published online: 24 September 2020

\section{References}

1. Laing, S. et al. Surface-enhanced Raman spectroscopy for in vivo biosensing. Nat. Rev. Chem. 1, 0060 (2017).

2. Li, J. F. et al. Shell-isolated nanoparticle-enhanced Raman spectroscopy. Nature 464, 392-395 (2010).

3. Aikens, C. M. et al. The effect of field gradient on SERS. Nat. Photon. 7, 508-510 (2013).

4. Wu, D. Y. et al. Electrochemical surface-enhanced Raman spectroscopy of nanostructures. Chem. Soc. Rev. 37, 1025-1041 (2008).

5. Lim, D. K. et al. Highly uniform and reproducible surface-enhanced Raman scattering from DNA-tailorable nanoparticles with 1-nm interior gap. Nat. Nanotechnol. 6, 452-460 (2011).

6. Lombardi, J. R. et al. A unified view of surface-enhanced Raman scattering. Acc. Chem. Res. 42, 734-742 (2009).

7. Yamamoto, Y. S. et al. Why and how do the shapes of surface-enhanced Raman scattering spectra change? Recent progress from mechanistic studies. J. Raman. Spectrosc. 47, 78-88 (2016).

8. Zong, C. et al. Surface-enhanced Raman spectroscopy for bioanalysis: reliability and challenges. Chem. Rev. 118, 4946-4980 (2018).

9. Panneerselvam, R. et al. Surface-enhanced Raman spectroscopy: bottlenecks and future directions. Chem. Commun. 54, 10-25 (2018).

10. Shi, R. et al. Facing challenges in real-life application of surface-enhanced Raman scattering: design and nanofabrication of surface-enhanced Raman scattering substrates for rapid field test of food contaminants. J. Agric. Food Chem. 66, 6525-6543 (2018).

11. Feng, S. M. et al. Ultrasensitive molecular sensor using n-doped graphene through enhanced Raman scattering. Sci. Adv. 2, e1600322 (2016).

12. Cong, S. et al. Electrochromic semiconductors as colorimetric SERS substrates with high reproducibility and renewability. Nat. Commun. 10, 678 (2019).

13. Willets, K. A. Super-resolution imaging of SERS hot spots. Chem. Soc. Rev. 43 3854-3864 (2014).

14. Shi, X. et al. Enhanced water splitting under modal strong coupling conditions. Nat. Nanotechnol. 13, 953-958 (2018).

15. Ding, S. Y. et al. Nanostructure-based plasmon-enhanced Raman spectroscopy for surface analysis of materials. Nat. Rev. Mater. 1, 16021 (2016).

16. Brolo, A. G. Plasmonics for future biosensors. Nat. Photon 6, 709-713 (2012).

17. Itoh, T. et al. Plasmon-enhanced spectroscopy of absorption and spontaneous emissions explained using cavity quantum optics. Chem. Soc. Rev. 46, 3904-3921 (2017).

18. Caldarola, M. et al. Non-plasmonic nanoantennas for surface enhanced spectroscopies with ultra-low heat conversion. Nat. Commun. 6, 7915 (2015).

19. Evlyukhin, A. B. et al. Demonstration of magnetic dipole resonances of dielectric nanospheres in the visible region. Nano Lett. 12, 3749-3755 (2012).

20. Wells, S. M. et al. Silicon nanopillars for field-enhanced surface spectroscopy. ACS Nano 6, 2948-2959 (2012).

21. He, Y. et al. Silicon nanowires-based highly-efficient SERS-active platform for ultrasensitive DNA detection. Nano Today 6, 122-130 (2011).

22. Ling, X. et al. Raman enhancement effect on two-dimensional layered materials: graphene, h-BN and $\mathrm{MoS}_{2}$. Nano Lett. 14, 3033-3040 (2014).

23. Park, W. H. et al. Out-of-plane directional charge transfer-assisted chemical enhancement in the surface-enhanced Raman spectroscopy of a graphene monolayer. J. Phys. Chem. C. 120, 24354-24359 (2016).

24. Zheng, Z. H. et al. Semiconductor SERS enhancement enabled by oxygen incorporation. Nat. Commun. 8, 1993 (2017). 
25. Musumeci, A. et al. SERS of semiconducting nanoparticles $\left(\mathrm{TiO}_{2}\right.$ hybrid composites). J. Am. Chem. Soc. 131, 6040-6041 (2009).

26. Limo, M. J. et al. Interactions between metal oxides and biomolecules: from fundamental understanding to applications. Chem. Rev. 118, 11118-11193 (2018).

27. Djurisic, A. B. et al. Toxicity of metal oxide nanoparticles: mechanisms, characterization, and avoiding experimental artefacts. Small 11, 26-44 (2015).

28. Chen, N. et al. Electronic logic gates from three-segment nanowires featuring two p-n heterojunctions. NPG Asia Mater. 5, e59 (2013).

29. Chiu, L. et al. Protein expression guided chemical profiling of living cells by the simultaneous observation of Raman scattering and anti-Stokes fluorescence emission. Sci. Rep. 7, 43569 (2017).

30. Shafer-Peltier, K. E. et al. Toward a glucose biosensor based on surfaceenhanced Raman scattering. J. Am. Chem. Soc. 125, 588-593 (2003).

31. Wang, J. Electrochemical glucose biosensors. Chem. Rev. 108, 814-825 (2008).

\section{Acknowledgements}

This research was supported by JSPS Core-to-Core Program, JSPS KAKENHI (JP18K13798, JP20K14785), Murata Science Foundation, MEXT Q-LEAP Program, White Rock Foundation, University of Tokyo GAP Fund, National Natural Science Foundation of China $(21671020,61805175)$, Beijing Natural Science Foundation (2172049), KISTEC, Nakatani Foundation, and Ogasawara Foundation for the Promotion of Science and Engineering.

\section{Author contributions}

N.C. and T.H.X. are co-first authors and contributed equally to this work. N.C., Z.L., and T.H.X. conducted the experiments. N.C., T.H.X., T.I., N.K., and K.G. conducted the theoretical calculations. N.C., T.H.X., Y.K., K.H., Z.C., and K.G. interpreted the data. Z.C. led the experimental work in the lab. T.H.X. and K.G. led the theoretical work. K.G. supervised the research team. N.C., T.H.X., Z.C., and K.G. wrote the paper. All authors discussed the results and participated in revising the paper.

\section{Competing interests}

N.C., T.H.X., Z.C., and K.G. are inventors on a pending patent that covers the design, fabrication, and use of the PCNA for SERS. The authors declare no other competing interests.

\section{Additional information}

Supplementary information is available for this paper at https://doi.org/10.1038/s41467020-18590-7.

Correspondence and requests for materials should be addressed to T.-H.X. or K.G.

Peer review information Nature Communications thanks the anonymous reviewers for their contribution to the peer review of this work. Peer reviewer reports are available.

Reprints and permission information is available at http://www.nature.com/reprints

Publisher's note Springer Nature remains neutral with regard to jurisdictional claims in published maps and institutional affiliations.

(c) (i) Open Access This article is licensed under a Creative Commons Attribution 4.0 International License, which permits use, sharing, adaptation, distribution and reproduction in any medium or format, as long as you give appropriate credit to the original author(s) and the source, provide a link to the Creative Commons license, and indicate if changes were made. The images or other third party material in this article are included in the article's Creative Commons license, unless indicated otherwise in a credit line to the material. If material is not included in the article's Creative Commons license and your intended use is not permitted by statutory regulation or exceeds the permitted use, you will need to obtain permission directly from the copyright holder. To view a copy of this license, visit http://creativecommons.org/ licenses/by/4.0/.

(C) The Author(s) 2020 